\title{
Nerve Stimulator vs Ultrasound-guided Femoral Nerve Block for Ease of Positioning before Spinal Anesthesia in Fracture Femur Patients: A Randomized Comparative Study
}

\author{
Avneet Janagal ${ }^{1}$, Geetanjali Pushkarna ${ }^{2}$, Ruchi Gupta ${ }^{3}$
}

\begin{abstract}
Introduction: Severe pain in the fractured femur makes it difficult to position a patient for spinal anesthesia. Femoral nerve block (FNB) has been studied for relief of pain, but studies are inconclusive regarding the superiority of one technique over another, i.e., nerve stimulation vs ultrasound for guidance.

Aims and objectives: This study aims to compare the ease of positioning for spinal anesthesia in fracture femur cases using two different techniques of localization of femoral nerve for the block.

Materials and methods: In this prospective, randomized, single-blind study, 60 patients of fracture femur were allocated in two equal groups of nerve stimulator (group NS) and ultrasound-guided (group US) femoral nerves block and compared regarding ease of positioning, duration of analgesia, and need for rescue doses of tramadol using SPSS version 26.

Results: The patients in the two groups were similar in age, sex, weight, BMl, and initial pain severity $(p=0.920)$. The visual analog scale (VAS) scores within the groups were markedly reduced at 15 minutes in both the groups $(p=0.000)$ and between the two groups pain relief was more in group US than group NS at 5 and 10 minutes ( $p=0.000$ and $p=0.034$, respectively), but, was comparable at 15 minutes $(p=0.310)$. The ease of positioning was observed in 27 patients in group US vs 20 patients NS ( $p=0.028$ ) as grade-I (comfortable), whereas grade II (discomfort) present in 3 vs 9 patients $(p=0.053)$, and grade III (uncomfortable) was seen only in one patient in group NS. The duration of analgesia in group US vs NS was $464.0 \pm 170.61$ and $282.9 \pm 126.85$ minutes, respectively $(p=0.003)$ and lesser need for rescue dose of tramadol (134.20 \pm 23.20 vs $174.43 .83 \pm 47.74 \mathrm{mg})(p=0.002)$. The vascular puncture was the main complication observed in group NS.

Conclusion: Both the techniques of FNB provided adequate analgesia for fracture femur pain, but the ultrasound technique provided earlyonset, better patient comfort, and longer duration of analgesia than the landmark nerves stimulation technique.

Keywords: Femoral nerve, Femoral nerve block, Fracture femur, Nerve stimulation, Pain, Ultrasound.

AMEI's Current Trends in Diagnosis \& Treatment (2020): 10.5005/jp-journals-10055-0105
\end{abstract}

\section{INTRODUCTION}

Fracture femur pain is a painful condition that often gets intolerable on movement especially during shifting, positioning during regional anesthesia or radiological evaluation. ${ }^{1}$ Various drugs are used for its management, such as nonsteroidal anti-inflammatory drugs or intravenous opioids, but, each has its limitations and side effects. Recently, femoral nerve block (FNB) which can be given either by using a nerve stimulator or under ultrasound guidance, has gained popularity. ${ }^{2,3}$ The peripheral nerve stimulation (PNS) had been the gold standard technique before the advent of ultrasound-guided (USG) nerve blocks, for adequate nerve determination during regional blocks. Ultrasound technique offers better pain relief perioperatively, lowers the dose and volume of local anesthetic with better localization of anatomical structures, and lesser use of systemic analgesics. But, the studies that compared both these techniques for fracture femur surgeries are limited, moreover, these studies had a few patients to prove the superiority of one technique over the other. So, this study intends to compare ease of positioning after femoral nerve block with ropivacaine $0.5 \%$ using nerve stimulator vs ultrasound technique before positioning for spinal anesthesia in fracture femur patients as a primary outcome. Various other parameters studied are the duration of analgesia and the amount of rescue analgesia requirement as a secondary outcome.
${ }^{1-3}$ Department of Anaesthesia, Sri Guru Ram Das Institute of Medical Sciences and Research, Amritsar, Punjab, India

Corresponding Author: Ruchi Gupta, Department of Anaesthesia, Sri Guru Ram Das Institute of Medical Sciences and Research, Amritsar, Punjab, India, Phone: +91 9814320805, e-mail: drruchisgrd@gmail. com

How to cite this article: Janagal A, Pushkarna G, Gupta R. Nerve Stimulator vs Ultrasound-guided Femoral Nerve Block for Ease of Positioning before Spinal Anesthesia in Fracture Femur Patients: A Randomized Comparative Study. AMEl's Curr Trends Diagn Treat 2020;4(2):78-83.

Source of support: Nil

Conflict of interest: None

\section{Materials and Methods}

This prospective, randomized, single-blind, and interventional study had been conducted after approval from the hospital ethics committee (SGRD/Patho/EC 201/19 dated 27/2/19) on 60 adult patients of either sex, weighing $50-80 \mathrm{~kg}$, belonging to the American Society of Anesthesiologist (ASA) grade I and II undergoing surgery for fracture femur surgery for one year. A prior written informed consent was obtained before starting the trial. 
Randomization was done using a computer-generated random number table, the numbers thus generated were categorized and sealed in the envelope. The slip from this envelope was taken out by the regional anesthesia team and the technique was decided according to the coded slip to which the assessor had been blinded. There was a single assessor throughout the study and she came into the operating room after the procedure got completed by the team. USG machine was kept for every patient but on standby mode for the PNS group, to avoid patient bias. The volume and concentration of ropivacaine were kept constant for all the patients to avoid further bias.

\section{Group NS $(n=30)$}

Patients in this group received $20 \mathrm{~mL}$ of $0.5 \%$ ropivacaine for femoral nerve block under nerve stimulator guidance.

\section{Group US ( $n=30)$}

Patients in this group received $20 \mathrm{~mL}$ of $0.5 \%$ ropivacaine for femoral nerve block under ultrasound guidance.

Patients with a known history of allergy, sensitivity, or any kind of reaction to local anesthetic agents, contraindication to neuraxial blockade (less platelet count, significant neurological disease, diagnosed increased intracranial pressure, epidural lipomatosis, tumors, fractured spine, and cauda equina syndrome), bleeding disorders, large inguinal lymph nodes or tumor at the site of injection, psychiatric or uncooperative, obese, pregnant, or kyphoscoliosis patients were excluded from the study.

A pre-anesthetic evaluation and routine investigations were done as per the protocol. After fasting for 6 hours, tablet alprazolam $0.5 \mathrm{mg}$ with sips of water was given as premedication. For administration of fluids and drugs, an intravenous line was established. Monitors like a pulse oximeter, noninvasive blood pressure (NIBP), and electrocardiography (ECG) are attached to the patients. Preoperative VAS was prior explained to the patient in the vernacular language, and evaluated on a scale of score $0-10$; with zero being no pain and 10 as the maximum pain one can experience. It was designated as V1.

The patient was made to lie in the supine position. The affected leg abducted and painted with $5 \%$ povidone-iodine. The inguinal ligament was identified and marked. The femoral artery was palpated and marked. A nerve stimulating needle of 22G (Stimuplex, ultra 360, B. Braun, Melsungen AG, Germany) was inserted at a $45^{\circ}$ just lateral the femoral artery. The current was initially started at $2 \mathrm{mV}$ using a nerve stimulator (Stimuplex HNS12, B. Braun, Melsungen AG, Germany), and then after checking the proper response of the quadriceps muscle contraction, it was reduced to $0.3-0.5 \mathrm{mV}$ (minimum current with which muscle contraction still visible).

Ultrasonography-guided FNB was given with a high-frequency linear probe (4-12 MHz) (InnoSight, Philips, USA) placed in the middle of the inguinal ligament, and after setting the optimal gain, depth, and focal point of the ultrasound femoral artery got visualized. The probe was then moved laterally, medially, and back and forth till a clear femoral nerve seen as a hyperechoic triangular-shaped structure immediately lateral to the femoral artery. Using the in-plane technique, the needle was inserted from the lateral side until it reached the femoral nerve. After identifying the nerve using either of the study techniques, a local anesthetic was administered after negative aspiration for blood. In the ultrasound method, we could see the spread of the drug around the nerve.

After performing a femoral nerve block, VAS was evaluated again after 5 and 10 minutes, designated as V2 and V3. Finally, assessed just before giving spinal anesthesia (V4). Ease of positioning for spinal anesthesia was graded as I, comfortable; II, discomfort; and III, uncomfortable.

The spinal anesthesia was performed under strict aseptic conditions at the level of L3-L4 intervertebral space. Then $2.5-3 \mathrm{~mL}$ of $0.5 \%$ bupivacaine injected after obtaining a free and clear flow of CSF. The time to perform spinal anesthesia was recorded. The highest level of sensory block determined in the mid-clavicular line bilaterally, checked by pinprick test using a $26-G$ hypodermic needle every 2 minutes till the level got stabilized for two consecutive readings 5 minutes apart. After ensuring an adequate level of block, surgery was allowed to start. Patients in both groups received oxygen at the rate of 4-6 L/min via face mask.

If no reduction in VAS even after 20 minutes of the femoral nerve block or before giving spinal anesthesia, then these patients were categorized into the failed block. If the effect of spinal anesthesia remained partial or worn off intraoperatively, then general anesthesia or total intravenous anesthesia was supplemented. All these patients were included in the randomization process, but, excluded from the outcome results.

Postoperatively in the recovery room (PACU), pulse rate, blood pressure, and $\mathrm{SpO}_{2}$ were measured. VAS was evaluated at 30 minutes intervals for the first 2 hours. In the ward, parameters recorded were pulse rate, blood pressure, and VAS at every 2 hours intervals for 6 hours (i.e., at 2, 4, 6, and 8 hours) and then at 12 and 24 hours. The duration of analgesia was taken from the sensory onset of a femoral nerve block to postoperative VAS $\geq 4$, then tramadol $2 \mathrm{mg} / \mathrm{kg}$ supplemented intravenously. The total number of boluses of tramadol received by the patient in 24 hours and the total dose was evaluated.

Complications of the femoral nerve block (vascular puncture, skin bruising, or femoral nerve injury), spinal anesthesia (postdural puncture headache, hypotension, bradycardia, and urinary retention), and toxicity of local anesthetic drugs or adverse effects of tramadol (nausea and vomiting) were observed and treated accordingly.

The sample size was determined based on the pilot study, using a $20 \%$ difference in VAS score for ease of positioning in both the groups. A total of 60 patients were included in the study with 30 patients in each group.

The data from this study was collected, decoded, compiled, and statistically analyzed with SPSS version 26 to draw relevant conclusions. The observations were tabulated in the form of mean \pm standard deviation (SD). For parametric data student's $t$-test, and for categorical data, a Chi-square test was applied. The level of significance was determined as its $p<0.05$ as significant and $p=0.001$ as highly significant.

\section{Results}

Data were analyzed for 29 patients in group NS and 30 patients in group US (Flowchart 1). Both the groups were comparable in terms of age, gender, ASA status, height, weight, BMI, and duration of surgery (Table 1).

The baseline VAS score was similar in both the groups, which decreased more in group US as compared to group NS at 5 and 10 minutes after the administration of FNB ( $p=0.000$ and 0.034 , respectively), but became similar between the groups at 15 minutes post-block $(p=0.310)$. However, VAS from baseline was markedly reduced within both the groups at all time intervals, indicating the overall efficacy of the procedure $(p<0.001)$ (Table 2). Ease of 
Flowchart 1: Consort flow diagram

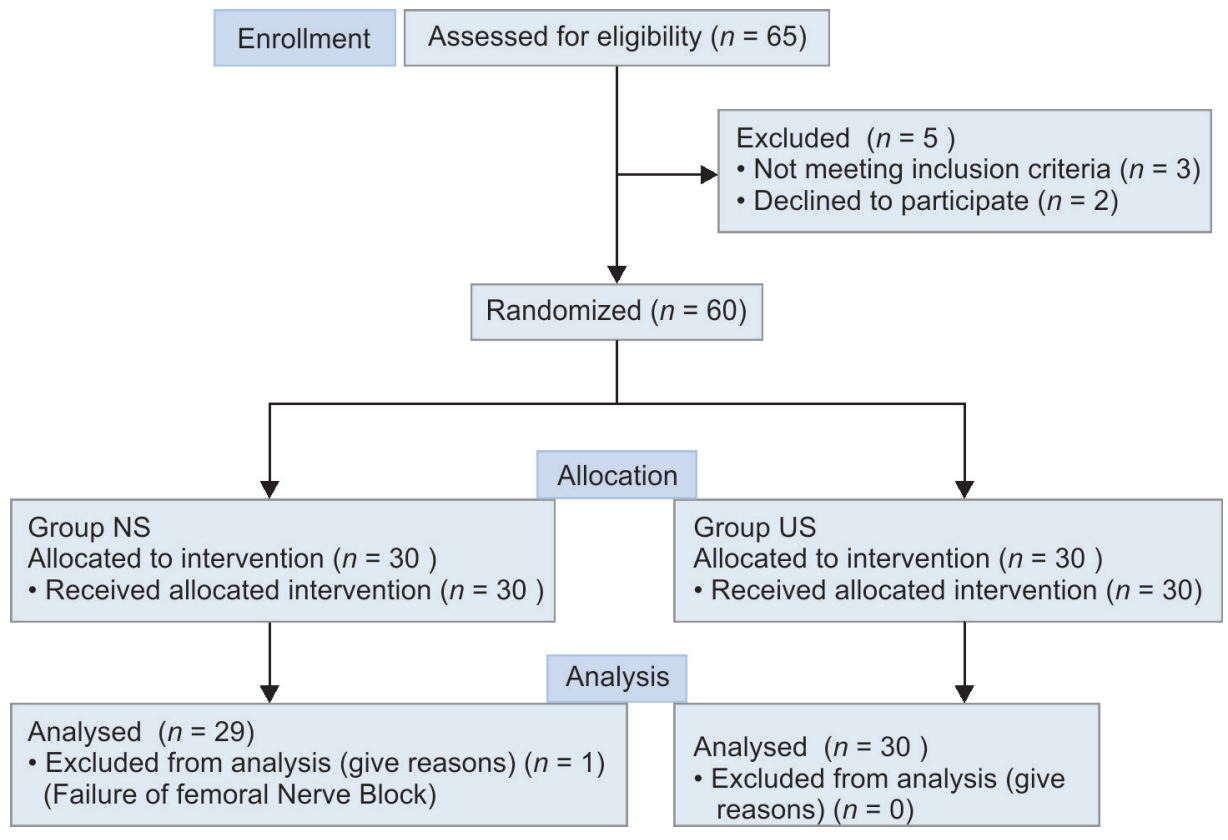

Table 1: Demographics of patients in two groups

\begin{tabular}{lcll}
\hline & Group NS $(n=30)$ & Group US $(n=30)$ & -value \\
\hline Age (years) & $49.17 \pm 19.64$ & $49.53 \pm 16.85$ & 0.938 \\
Gender (M/F) & $19 / 11$ & $17 / 13$ & 0.598 \\
ASA (I/II) & $15 / 15$ & $13 / 17$ & 0.605 \\
Height $(\mathrm{cm})$ & $153.43 \pm 1.48$ & $152.8 \pm 1.69$ & 0.128 \\
Weight $(\mathrm{kg})$ & $64.5 \pm 4.27$ & $64.83 \pm 7.13$ & 0.827 \\
BMI $\left(\mathrm{kg} / \mathrm{m}^{2}\right)$ & $27.43 \pm 2.11$ & $27.9 \pm 3.11$ & 0.499 \\
Duration of surgery & $114 \pm 11.02$ & $112.67 \pm 10.48$ & 0.633 \\
(min) & & & \\
\hline
\end{tabular}

Data are mean $\pm S D ; n$, number of patients

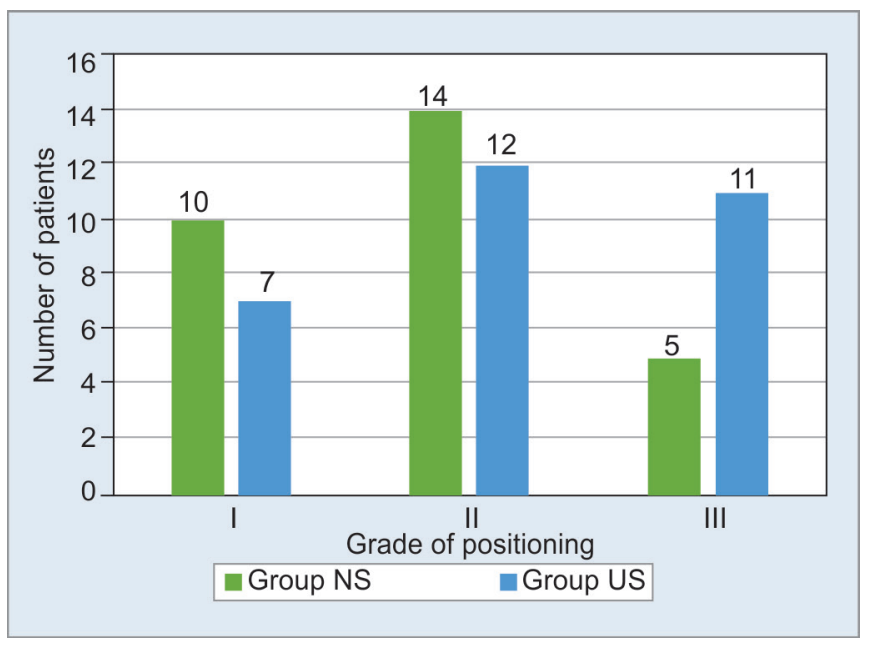

Fig. 1: Grades of ease of positioning in two groups

Table 2: Visual analog scale at different time intervals after the femoral nerve block

\begin{tabular}{|c|c|c|c|c|c|c|c|}
\hline \multirow[b]{2}{*}{ VAS (min) } & \multicolumn{3}{|c|}{ Group NS $(n=30)$} & \multicolumn{3}{|c|}{ Group US $(n=30)$} & \multirow[b]{2}{*}{$p$-value } \\
\hline & Mean $\pm S D$ & Mean difference & w.r.b & Mean $\pm S D$ & Mean difference & w.r.b & \\
\hline 0 & $61.38 \pm 15.05$ & - & - & $61.67 \pm 3.79$ & - & - & 0.920 \\
\hline 5 & $53.45 \pm 8.14$ & 7.93 & 0.018 & $46 \pm 6.21$ & 15.67 & 0.000 & 0.000 \\
\hline 10 & $35.86 \pm 7.33$ & 25.52 & 0.000 & $31.67 \pm 7.47$ & 30.00 & 0.000 & 0.034 \\
\hline 15 & $21.38 \pm 7.89$ & 40.00 & 0.000 & $18.67 \pm 11.96$ & 43.00 & 0.000 & 0.310 \\
\hline
\end{tabular}

w.r.b, with respect to baseline; $n$, number of patients

positioning was graded as I, II, and III, most of the patients 27 (94\%) in group US were comfortable (grade I), probably due to early and good pain relief, followed by $3(10 \%)$ patients with grade II, and none with grade III, vs grade I in 20 (67\%) patients in group NS, 9 (30\%) patients had some discomfort (grade II), and 1 (3\%) had severe pain with uncomfortable posture (grade III) (Fig. 1).
Intraoperatively, hemodynamic parameters were comparable between the groups. There were similar numbers of patients in both the groups who had hypotension or bradycardia, probably due to the effect of spinal anesthesia (Fig. 2).

The duration of postoperative analgesia in group NS was $282.9 \pm 126.85$ minutes and in group US was $464.0 \pm 170.61$ minutes 

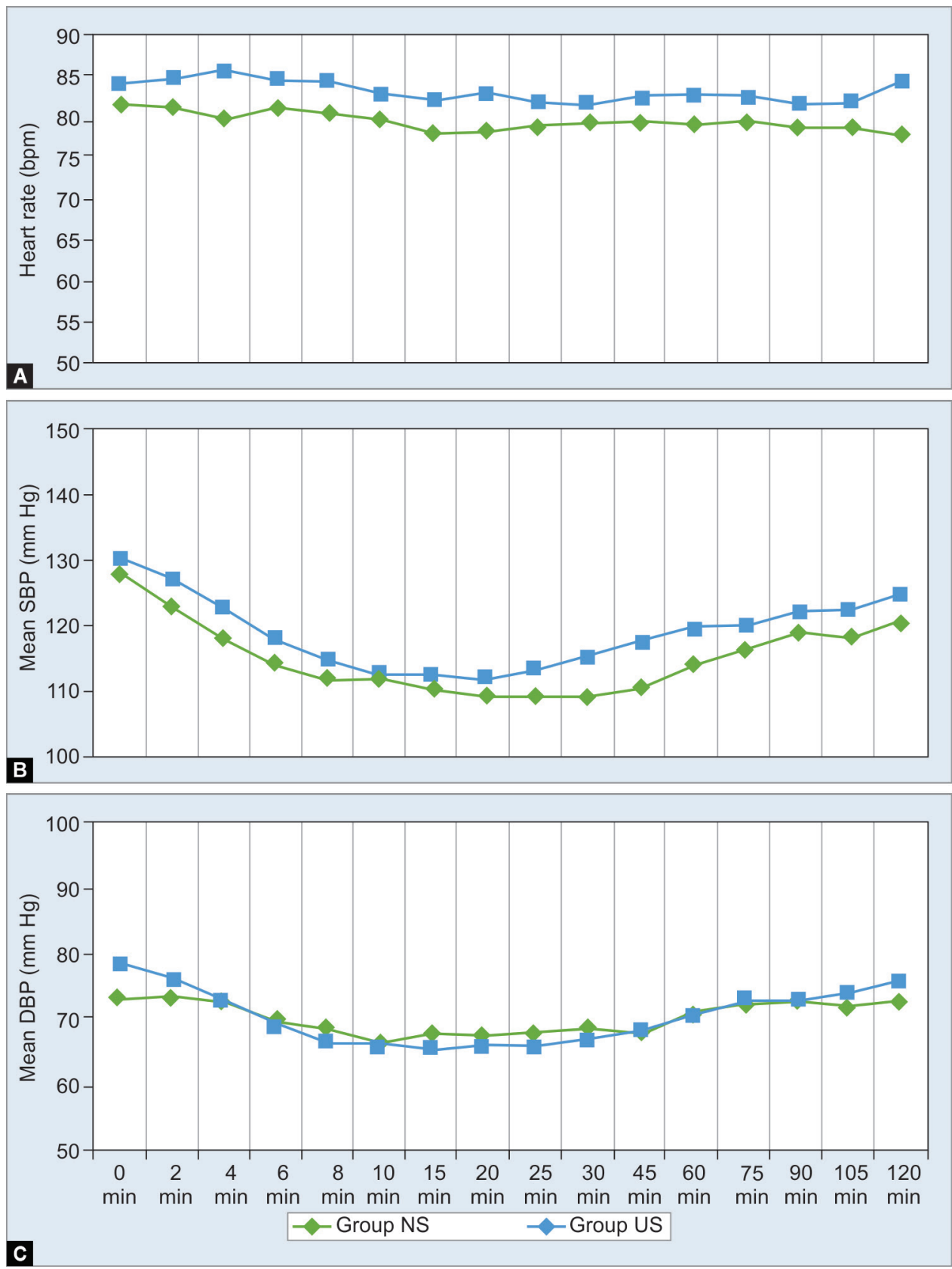

Figs $2 \mathrm{~A}$ to $\mathrm{C}$ : Intraoperative heart rate, systolic blood pressure (SBP), and diastolic blood pressure (DBP) in two groups

( $p=0.003$ ) (Fig. 3). Because of this difference in duration of postoperative analgesia, even the 24-hour requirement of tramadol was more in group NS, i.e.,174.43 $\pm 47.74 \mathrm{mg}$, compared to $134.20 \pm 23.20 \mathrm{mg}$ in group US ( $p=0.002$ ) (Fig. 4).

Complications, such as vascular puncture and skin bruising were more in the NS group because of its blind technique, whereas other minor complications were similar between the two groups. There was no case of nerve injury observed in any case (Fig. 5).

\section{Discussion}

Movement and positioning of the patients with fracture femur before regional anesthesia is extremely painful as evident from preoperative baseline VAS scores as observed in our study and described in other studies. ${ }^{1,4}$ Paracetamol, ketamine, non-steroidal anti-inflammatory drugs, or opioids have been used to relieve pain during positioning, but have their limitations. ${ }^{2,3}$ The femoral nerve block, a simple and easy to perform procedure, has been used both pre-emptively as well as post-operatively for fracture femur surgeries, anterior aspect of thigh or knee surgeries, or for physiotherapy after knee surgery. ${ }^{2,3,5}$ Conventionally, landmark technique with a nerve stimulator (NS) has been used in the past to achieve precise localization of nerves with high efficiency. ${ }^{6,7} \mathrm{Liu}$ et al. attributed this success rate to the experience and expertise of anaesthesiologists. ${ }^{8}$ The blind exploration can sometimes damage nerves or vessels. However, with the advent of ultrasound 
Nerve Stimulator vs Ultrasound-guided Femoral Nerve Block

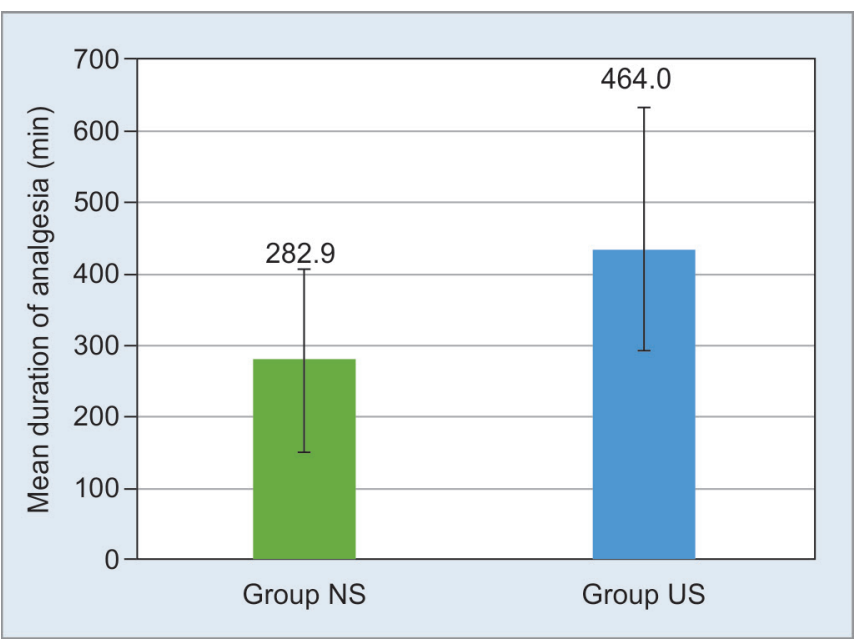

Fig. 3: Duration of analgesia (minutes) in two groups

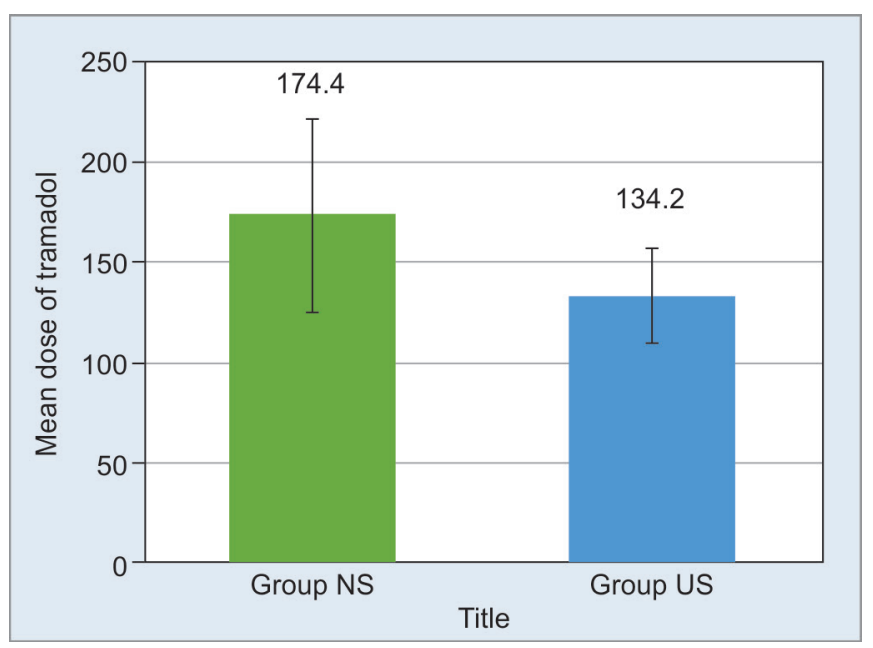

Fig. 4: Mean dose of tramadol (mg) in two groups

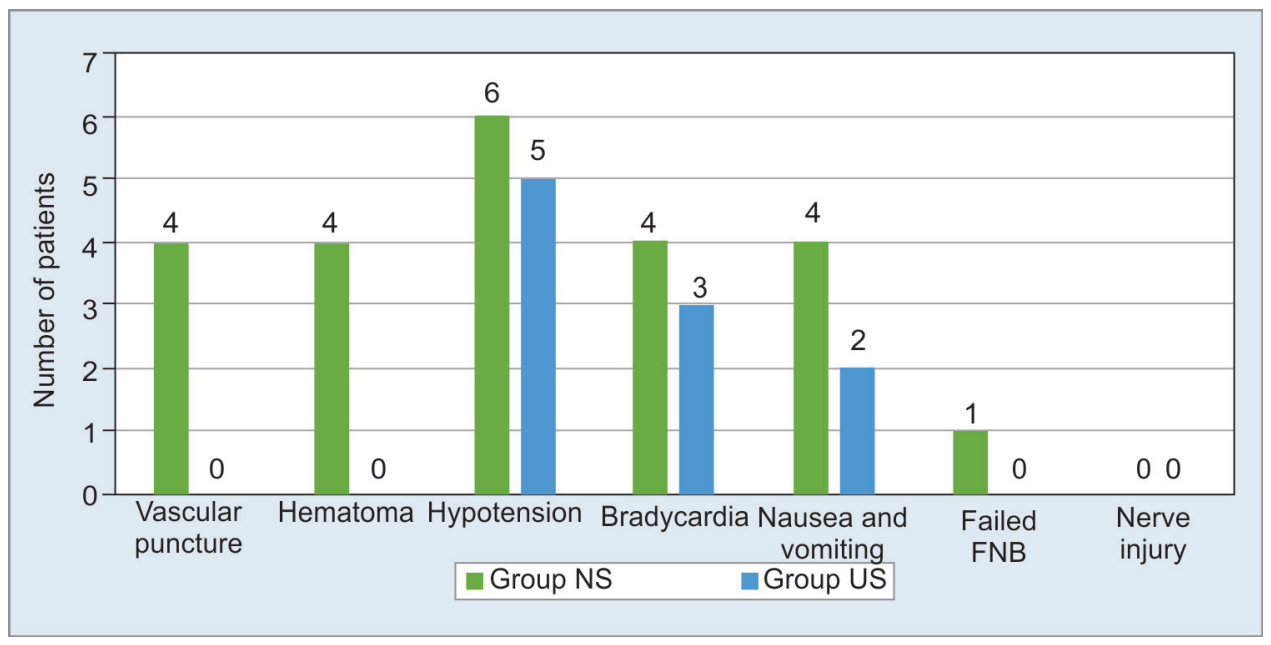

Fig. 5: Complications in two groups

(US) in anesthesia practice, US-guided nerve blocks have become technically more effective, safe, and easy to perform procedures. ${ }^{2}$ One can visualize needle, nerve, surrounding structures, and spread of the local anesthetic agent. The only shortcoming regarding its use is its availability in resource-limited hospital settings and high cost. In a meta-analysis by Abrahams et al., US-guided blocks resulted in shorter procedure time, 29\% faster onset, $25 \%$ increase in duration and lesser vascular punctures as compared to peripheral nerve blocks performed using a nerve stimulator. 9

Gupta et al. reported significant VAS reduction after 5 minutes in a group receiving US-guided FNB in fracture femur surgeries. ${ }^{5}$ Similarly, in the present study we found earlier pain relief at 5 and 10 minutes in US-guided FNB as compared to NS-guided FNB. This was probably because of accurate drug deposition around the femoral nerve compared to blind placement in the case of group NS. However, by 15 minutes the VAS reduction was comparable between the groups. This signifies early onset with the US-guided FNB technique. Forouzan et al., found the success rate of the NS technique to be $92 \%$ at 30 minutes as compared to $100 \%$ with the US technique of FNB in fracture femur patients. ${ }^{10}$
Rubin et al. showed the time of onset to be significantly lower with ultrasound than nerve stimulation in children. ${ }^{11}$ Pain relief with FNB in fracture femur patients could be due to deposition of ropivacaine within close vicinity of femoral nerve under US guidance which increases the chances of articular branches getting blocked. ${ }^{4}$

Most of the patients reported the ease of positioning as comfortable in both the groups after FNB, but, the number of patients with grade I ease was significantly more in group US compared to group NS, again due to better pain relief. Few patients in group NS complained of some discomfort while being positioned for spinal anesthesia compared to group US. One patient who had severe pain and uncomfortable positioning in group NS was considered block failure and excluded from the results.

The prolonged duration of analgesia and the lesser requirement of a total dose of tramadol in the US-guided technique further prove that deposition of the local anesthetic in close proximity to the nerve affects the outcome. Our results have been similar to Jain et al. who used $0.5 \%$ ropivacaine for FNB. ${ }^{4}$ The duration of analgesia with US-guided FNB was 5.2 hours in the study by 
Gupta et al., whereas we had 7.7 hours, the difference may be due to the use of different local anaesthetics. ${ }^{5}$ Singh et al. found the duration of analgesia to be 6 hours with nerve stimulating technique of FNB using $0.2 \%$ ropivacaine, whereas it was 4.7 hours in our study. ${ }^{12}$ This may be due to the additive effect of $0.75 \%$ ropivacaine used intra-spinally and top-ups of $0.2 \%$ ropivacaine used intraoperatively by them.

Complications observed due to FNB in our study were mainly vascular puncture and skin bruising in the case of NS technique due to blind execution. Various authors have quoted the incidence of vascular puncture to vary between $15 \%$ and $56.4 \%$ after nerve stimulation technique of peripheral nerve blocks vs $5 \%$ by ultrasound modality. ${ }^{13-16}$ NS technique does not guarantee avoidance of intraneural placement of the needle. Nerve injuries have also been reported without major adverse sequelae even with ultrasound technique by Schafhalter-Zoppoth et al., but, we did not have any such complication in any patient in our study. ${ }^{17}$

Both nerve stimulation and ultrasound technique of FNB has its advantages and disadvantages. Both have a learning curve and are safe and easy in the hands of an experienced practitioner, therefore, one may decide to use either of them or as a combined technique for more affectivity.

\section{Limitation of OUR Study}

Parameters for ease of positioning were subjectively evaluated rather than objective assessment. The blocks performed were by the junior faculty, so the experience might have made some difference in a number of complications.

To conclude, femoral nerve block provided effective analgesia during positioning of patients for spinal anesthesia undergoing fracture femur surgery. But, an ultrasound-guided technique could be performed safely with better patient comfort and longer duration of analgesia than landmark nerves stimulation technique.

\section{References}

1. Somvanshi M, Tripathi A, Meena N. Femoral nerve block for acute pain relief in fracture shaft femur in an emergency ward. Saudi J Anaesth 2015;9(4):439-441. DOI: 10.4103/1658-354X.159471.

2. Christos SC, Chiampas G, Offman R, et al. Ultrasound-guided three-in-one nerve block for femur fractures. West J Emerg Med 2010;11(4):310-313. PMID: 21079698, PMCID: PMC2967678.

3. Wang F, Liu LW, Hu Z, et al. Ultrasound, and nerve stimulator guided continuous femoral nerve block analgesia after total knee arthroplasty: a multicenter randomized controlled study. Rev Bras Anestesiol 2015;65(1):14-20. DOI: 10.1016/j.bjan.2013.07.007.

4. Jain N, Mathur PR, Patodi V, et al. A comparative study of ultrasoundguided femoral nerve block versus fascia iliaca compartment block in patients with fracture femur for reducing pain associated with positioning for subarachnoid block. Indian J Pain 2018;32(3):150-154. DOI: 10.4103/ijpn.ijpn_21_18.

5. Gupta M, Kamath SS. Comparison of preoperative ultrasound guided fascia iliaca block versus femoral nerve block for proximal femur fractures before positioning for spinal anesthesia: an observational study. Korean J Pain 2020;33(2):138-143. DOI: 10.3344/kjp.2020.33.2.138.

6. Fanelli G, Casati A, Garancini P, et al. Nerve stimulator and multiple injection technique for upper and lower limb blockade: failure rate, patient acceptance, and neurologic complications. Study Group on Regional Anesthesia. Anesth Analg 1999;88(4):847-852. DOI: 10.1213/00000539-199904000-00031.

7. Franco $C D$, Vieira ZE. 1,001 subclavian perivascular brachial plexus blocks: success with a nerve stimulator. Reg Anesth Pain Med 2000;25(1):41-46. DOI: 10.1016/s1098-7339(00)80009-7.

8. Liu SS, Zayas VM, Gordon MA, et al. A prospective, randomized, controlled trial comparing ultrasound versus nerve stimulator guidance for interscalene block for ambulatory shoulder surgery for postoperative neurological symptoms. Anesth Analg 2009;109(1):265271. DOI: 10.1213/ane.0b013e3181a3272c.

9. Abrahams MS, Aziz MF, Fu RF, et al. Ultrasound guidance compared with electrical neurostimulation for peripheral nerve block: a systematic review and meta-analysis of randomised controlled trials. Br J Anaesth 2009;102(3):408-417. DOI: 10.1093/bja/aen384.

10. Forouzan A, Masoumi K, Motamed H, et al. Nerve stimulator versus ultrasound-guided femoral nerve block; a randomised clinical trial. Emerg (Tehran) 2017;5(1):e54. PMID: 28286861, PMCID: PMC5325926.

11. Rubin K, Sullivan D, Sadhasivam S. Are peripheral and neuraxial blocks with ultrasound guidance more effective and safer in children? Paediatr Anaesth 2009;19(2):92-96. DOI: 10.1111/j.14609592.2008.02918.x.

12. Singh AP, Kohli V, Bajwa SJ. Intravenous analgesia with opioids versus femoral nerve block with $0.2 \%$ ropivacaine as preemptive analgesic for fracture femur: a randomized comparative study. Anesth Essays Res 2016;10(2):338-342. DOI: 10.4103/0259-1162.176403.

13. Marhofer $\mathrm{P}$, Schrögendorfer $\mathrm{K}$, Koinig $\mathrm{H}$, et al. Ultrasonographic guidance improves sensory block and onset time of three-in-one blocks. Anesth Analg 1997;85(4):854-857. DOI: 10.1097/00000539199710000-00026.

14. Marhofer $P$, Schrögendorfer $K$, Wallner $T$, et al. Ultrasonographic guidance reduces the amount of local anesthetic for 3-in-1 blocks. Reg Anesth Pain Med 1998;23(6):584-548. DOI: 10.1016/s10987339(98)90086-4.

15. Liu FC, Liou JT, Tsai YF, et al. Efficacy of ultrasound-guided axillary brachial plexus block: a comparative study with nerve stimulatorguided method. Chang Gung Med J 2005;28(6):396-402. PMID: 16124155.

16. Sauter AR, Dodgson MS, Stubhaug A, et al. Electrical nerve stimulation or ultrasound guidance for lateral sagittal infraclavicular blocks: a randomized, controlled, observer-blinded, comparative study. Anesth Analg 2008;106(6):1910-1915. DOI: 10.1213/ane.0b013e318173280f.

17. Schafhalter-Zoppoth I, Gray AT. Inadvertent femoral nerve impalement and intraneural injection visualized by ultrasound. Anesth Analg 2004;99(2):627-628. DOI: 10.1213/01.ANE.0000130913. 31395.40 . 